\title{
Long non-coding RNA NEAT1 promotes migration and invasion of oral squamous cell carcinoma cells by sponging microRNA-365
}

\author{
XIAOHUA LIU, WENZHI SHANG and FUJU ZHENG \\ Department of Endodontics, Jinan Stomatological Hospital, Jinan, Shandong 250001, P.R. China
}

Received January 2, 2018; Accepted May 25, 2018

DOI: $10.3892 /$ etm.2018.6493

\begin{abstract}
Long non-coding RNA nuclear enriched abundant transcript 1 (NEAT1) has been demonstrated to serve key roles in numerous human cancer types, but its function in oral squamous cell carcinoma (OSCC) and underlying regulatory mechanism have not been evaluated. The present study demonstrated that expression of NEAT1 was significantly higher in OSCC tissue and cell lines compared with adjacent non-tumour tissue and normal oral keratinocytes, respectively. Additionally, upregulation of NEAT1 was significantly associated with advanced clinical stage and shorter survival time in patients with OSCC. Bioinformatics analysis and luciferase reporter gene assay data confirmed the interaction between NEAT1 and miR-365, and it was revealed that NEAT1 may downregulate microRNA (miR)-365 expression in OSCC cells. Furthermore, inhibition of NEAT1 expression led to a significant reduction in OSCC cell migration and invasion, which was accompanied by reduced matrix metalloproteinase (MMP)-2 and MMP9 protein expression. By contrast, inhibition of miR-365 eliminated suppressive effects of NEAT1 knockdown on OSCC cell migration and invasion. miR-365 was significantly downregulated in OSCC tissue and cell lines and an inverse correlation between miR-365 and NEAT1 expression in OSCC tissue was observed. To conclude, the present study demonstrated that NEAT1 promoted migration and invasiveness of OSCC cells by sponging miR-365. The current study suggests that NEAT1 may serve as a novel therapeutic target for the treatment of OSCC.
\end{abstract}

\section{Introduction}

Oral squamous cell carcinoma (OSCC) is the most common malignancy of the oral cavity, accounting for $>90 \%$ of all oral

Correspondence to: Dr Xiaohua Liu, Department of Endodontics, Jinan Stomatological Hospital, 101 Jingliu Road, Jinan, Shandong 250001, P.R. China

E-mail: sdliuxiaohua@sina.com

Key words: nuclear enriched abundant transcript 1, microRNA, migration, invasion, oral squamous cell carcinoma cancer cases (1-3). Although substantial advances have been made in the treatment of OSCC, including surgical resection combined with chemotherapy and radiotherapy, the prognosis of patients with advanced OSCC is unsatisfactory $(4,5)$. Therefore, it is necessary to explore underlying mechanisms of OSCC tumourigenesis, which may be helpful in the development of novel therapeutic targets.

Long non-coding RNAs (lncRNAs) are a group of small and single-stranded non-coding RNAs that are $>200 \mathrm{nt}$ in size and evolutionarily conserved (6,7). Although lncRNAs exhibit no protein-coding capacity, they are key regulators of gene expression and serve important roles in various biological processes, including cell growth, migration, invasion and tumourigenesis $(8,9)$. In recent years, evidence has suggested that lncRNAs serve promoting or tumour suppressive roles in the development and malignant progression of OSCC $(10,11)$. For instance, lncRNA taurine upregulated gene 1 promotes progression of OSCC through upregulation of formin-like 2 by acting as molecular sponges for microRNA (miR)-219 (12). LncRNA maternally expressed 3 inhibits proliferation and metastasis of OSCC via regulation of the $\mathrm{WNT} / \beta$-catenin signalling pathway (13). Recently, IncRNA nuclear enriched abundant transcript 1 (NEAT1) has been observed to be upregulated in several human cancer types and studies have revealed its oncogenic role (14-16). For example, NEAT1 is regulated by the epidermal growth factor receptor signalling pathway and contributes to glioblastoma progression through the WNT/ $\beta$-catenin pathway by serving as a scaffold for enhancer of zeste homolog 2 (15). NEAT1 contributes to paclitaxel resistance in ovarian cancer cells through regulation of zinc finger E-box-binding homeobox 1 expression via miR-194 (16). However, to the best of our knowledge, the function of NEAT1 in OSCC has not yet been reported.

miRs are a class of small non-coding RNAs that are composed of $\sim 22-25 \mathrm{nt}$ and have been demonstrated to regulate gene expression via binding to the 3'-untranslated region (3'-UTR) of their target mRNAs, which results in mRNA degradation or translation repression $(17,18)$. Similar to lncRNAs, miRs are also involved in development and progression of tumours, including OSCC (2,3,17-19). Recently, miR-365 has been reported to serve a general tumour-suppressive role in multiple human cancer types; for instance, inhibiting ovarian cancer progression by targeting Wnt5a (20). Additionally, miR-365 inhibits proliferation, migration and invasiveness of 
glioma cells by targeting phosphoinositide-3-kinase regulatory subunit 3 (21). However, to the best of our knowledge, the exact role of miR-365 in OSCC has not been reported.

In addition, matrix metalloproteinase (MMP)2 and MMP9 are two key enzymes involved in tumour cell invasiveness (22). Whether these enzymes are involved in the function of NEAT1 and miR-365 in OSCC still remains unclear.

The present study aimed to investigate NEAT1 and miR-365 expression levels and functions in OSCC, as well as their underlying molecular mechanism in vitro.

\section{Materials and methods}

Clinical tissues. The current study was approved by the Research Ethics Committee of the Stomatological Hospital of Jinan City (Jinan, China). A total of 58 OSCC and adjacent non-tumour tissues were collected from patients with primary OSCC at the Stomatological Hospital of Jinan City between March 2010 and April 2012. No patient received chemotherapy or radiotherapy before surgical resection. The clinicopathological characteristics of the patients are summarized in Table I. Written informed consent was obtained from all patients. Tissues were immediately snap-frozen in liquid nitrogen and stored at $-80^{\circ} \mathrm{C}$ until use.

Cell culture and transfection. Human OSCC cell lines HN4, Tca-8113, UM-SCC-1, Cal-27, SCC-25 and SCCKN, and the normal human oral keratinocyte cell line hNOK were purchased from the Cell Bank of the Chinese Academy of Sciences (Shanghai, China). All cell lines were cultured in Dulbecco's modified Eagle's medium (DMEM; Thermo Fisher Scientific, Inc., Waltham, MA, USA) supplemented with $10 \%$ fetal bovine serum (FBS; Thermo Fisher Scientific, Inc.) at $37^{\circ} \mathrm{C}$ in humidified atmosphere with $5 \% \mathrm{CO}_{2}$. Lipofectamine 2000 (Thermo Fisher Scientific, Inc.) was used to transfect HN4 and Tca-8113 cells with $100 \mathrm{nM}$ of non-specific short interfering RNA (NC siRNA; Am10301; Amspring, Changsha, China), NEAT1-specific siRNA (NEAT1 siRNA; Am10542; Amspring), pcDNA3.1 vector (Am00013; Amspring), pcDNA-NEAT1 expression plasmid (Am02051; Amspring), miR-365 inhibitor (HmiR-AN0451-SN-10; Guangzhou Fulengen Co., Ltd., Guangzhou, China) and negative control (NC) inhibitor (CmiR-AN0001-SN; Guangzhou Fulengen Co., Ltd.) according to the manufacturer's instructions. Subsequent experiments were performed $48 \mathrm{~h}$ following transfection.

Reverse transcription-quantitative polymerase chain reaction $(R T-q P C R)$. Total RNA was extracted from tissues and cells using TRIzol reagent (Thermo Fisher Scientific, Inc.), while genomic DNA was removed by treatment with DNase (Thermo Fisher Scientific, Inc.) according to the manufacturer's instructions. Total RNA $(1 \mu \mathrm{g})$ was reverse transcribed using a RevertAid First Stand cDNA Synthesis kit (Thermo Fisher Scientific, Inc.) according to the manufacturer's instructions. Reverse transcription was performed at $16^{\circ} \mathrm{C}$ for $30 \mathrm{~min}$, followed by incubation at $42^{\circ} \mathrm{C}$ for $30 \mathrm{~min}$ and enzyme inactivation at $85^{\circ} \mathrm{C}$ for $5 \mathrm{~min}$. The expression of miR-365 was detected using miScript SYBR Green PCR kit (Qiagen, Inc., Valencia, CA, USA) on an ABI 7500 PCR machine (Thermo Fisher Scientific, Inc.). U6 was used as the internal reference. To detect NEAT1 expression, qPCR was performed using Power SYBR Green PCR Master mix (Thermo Fisher Scientific, Inc.). GAPDH was used as the internal reference. The reaction conditions for all qPCR experiments were as follows: $95^{\circ} \mathrm{C}$ for $5 \mathrm{~min}$, followed by 40 cycles of $95^{\circ} \mathrm{C}$ for $10 \mathrm{sec}, 60^{\circ} \mathrm{C}$ for $30 \mathrm{sec}$ and $72^{\circ} \mathrm{C}$ for $30 \mathrm{sec}$. The relative expression was determined using the $2^{-\Delta \Delta C q}$ method (23). The following primers were used: U6, forward 5'-CTCGCTTCGGCAGCACATATACT-3' and reverse 5'-CGCTTCACGAATTTGCGTGT-3'; GAPDH, forward 5'-GGAGCGAGATCCCTCCAAAAT-3' and reverse 5'-GGCTGTTGTCATACTTCTCATGG-3'. miR-365 primers were purchased from Guangzhou Fulengen Co., Ltd.

Bioinformatics analysis. Target genes for NEAT1 and miR-365 were predicted using RNAhybrid 2.12 (http://bibiserv.techfak. uni-bielefeld.de/rnahybrid/).

Luciferase reporter gene assay. Fragments of NEAT1 containing wild-type (WT) or mutant type (MT) miR-365 binding sites were cloned into pmirGLO Dual-luciferase Target Expression Vector (Promega Corporation, Madison, WI, USA), which generated WT or MT NEAT1 plasmids. HN4 and Tca-8113 cells were co-transfected with miR-365 mimic, scramble miR mimic (miR-NC), WT or MT NEAT1 plasmid using Lipofectamine 2000. Following $48 \mathrm{~h}$ of transfection, luciferase reporter gene assays were performed using the Dual-Luciferase Reporter Assay System (Promega Corporation). The firefly luciferase activity was normalized against Renilla luciferase activity.

Wound-healing assay. Wound-healing assays were conducted to determine the migratory capacity of cells. HN4 and Tca- 8113 cells were cultured to full confluence, wounds of $\sim 1 \mathrm{~mm}$ in width were generated with a plastic scriber and cells were washed with PBS. Cells were then cultured at $37^{\circ} \mathrm{C}$ with $5 \% \mathrm{CO}_{2}$ for $48 \mathrm{~h}$ and assessed with an inverted microscope (magnification, $\mathrm{x} 40$ ).

Transwell assay. HN4 and Tca-8113 cells $(10,000 /$ well $)$ in DMEM were added to the upper chamber of Transwell inserts, pre-coated with Matrigel (BD Biosciences, Franklin Lakes, NJ, USA) and DMEM supplemented with 10\% FBS was added to the lower chamber. HN4 and Tca-8113 cells were then incubated at $37^{\circ} \mathrm{C}$ for $24 \mathrm{~h}$. HN4 and Tca- 8113 cells that had not migrated through the membrane of the insert were removed using a cotton-tipped swab, while the cells on the lower surface of the membrane were stained with gentian violet (Sigma-Aldrich; Merck KGaA, Darmstadt, Germany) at room temperature for $10 \mathrm{~min}$ and counted under an inverted microscope (magnification, $\mathrm{x} 400$ ).

Western blotting. Tissues and cells were lysed in cold radioimmunoprecipitation assay buffer (Thermo Fisher Scientific, Inc.) and the protein concentration was determined using a Bicinchoninic Acid Protein Assay kit (Thermo Fisher Scientific, Inc.). Proteins (50 $\mu \mathrm{g}$ ) were separated on $10 \%$ SDS-PAGE gels and transferred to a polyvinylidene difluoride membrane (Thermo Fisher Scientific, Inc.). The membrane was blocked in 5\% non-fat milk in PBS containing $0.1 \%$ Tween-20 (Sigma-Aldrich; Merck KGaA) at room temperature for $3 \mathrm{~h}$. It was then incubated with rabbit polyclonal anti-human MMP2 
Table I. Association between NEAT1 expression and clinicopathological characteristics in patients with oral squamous cell carcinoma.

\begin{tabular}{|c|c|c|c|c|}
\hline \multirow[b]{2}{*}{ Variable } & \multirow[b]{2}{*}{ Number $(n=58)$} & \multicolumn{2}{|c|}{ NEAT1 expression } & \multirow[b]{2}{*}{ P-value } \\
\hline & & Low $(n=32)$ & High $(n=26)$ & \\
\hline Age (years) & & & & 0.791 \\
\hline$\leq 55$ & 24 & 14 & 10 & \\
\hline$>55$ & 34 & 18 & 16 & \\
\hline Sex & & & & 0.183 \\
\hline Male & 35 & 22 & 13 & \\
\hline Female & 23 & 10 & 13 & \\
\hline Differentiation grade & & & & 0.213 \\
\hline Well and moderately & 45 & 27 & 18 & \\
\hline Poor & 13 & 5 & 8 & \\
\hline Lymph node metastasis & & & & 0.009 \\
\hline Present & 18 & 5 & 13 & \\
\hline Absent & 40 & 27 & 13 & \\
\hline Tumor, node and metastasis stage & & & & 0.007 \\
\hline I-II & 36 & 25 & 11 & \\
\hline III-IV & 22 & 7 & 15 & \\
\hline
\end{tabular}

NEAT1, nuclear enriched abundant transcript 1.

(1:200; ab37150; Abcam, Cambridge, MA, USA), rabbit polyclonal anti-human MMP9 (1:200; ab38898; Abcam) or rabbit polyclonal anti-human GAPDH (1:100; ab9485; Abcam) at room temperature for $3 \mathrm{~h}$. This was followed by incubation with the horseradish peroxidase-conjugated goat anti-rabbit secondary IgG antibody (1:5,000; ab6721; Abcam) at room temperature for $1 \mathrm{~h}$. Enhanced chemiluminescence (Thermo Fisher Scientific, Inc.) was used to examine protein expression, which was analysed using Image-Pro Plus software 6.0 (Media Cybernetics, Inc., Rockville, MD, USA) according to the manufacturer's protocol.

Statistical analysis. All data are presented as the mean \pm standard deviation. SPSS 18.0 (SPSS, Inc., Chicago, IL, USA) was used for statistical analysis. Student's t-test was used for comparisons between two groups, while one-way analysis of variance followed by Tukey's post hoc test was used for comparisons of $>2$ groups. The associations between NEAT1 expression and the clinicopathological characteristics of OSCC were examined using the $\chi^{2}$ test. The Kaplan-Meier method was applied to analyse overall survival of patients with OSCC. Pearson's correlation was used to analyze the correlation between NEAT1 and miR-365 expression in OSCC tissues. $\mathrm{P}<0.05$ was considered to indicate a statistically significant difference.

\section{Results}

NEAT1 is upregulated in OSCC. In the present study, NEAT1 expression in OSCC and adjacent non-tumour tissue was evaluated. RT-qPCR assay data indicated that NEAT1 was significantly upregulated in OSCC tissue compared with adjacent non-tumour tissue (Fig. 1A). Based on the mean expression value of NEAT1 as cut-off value (5.54), these patients with OSCC were divided into high and low expression groups. Further investigation indicated that increased NEAT1 expression was significantly associated with positive lymph node metastasis and advanced clinical stage (Table I). NEAT1 was significantly upregulated in OSCC cell lines compared with hNOK cells (Fig. 1B). Additionally, patients with OSCC and high NEAT1 expression exhibited a shorter survival time compared with patients with low NEAT1 expression (Fig. 1C).

MiR-365 is downregulated in OSCC. Potential target miRs of NEAT1 in OSCC cells were evaluated. A bioinformatics analysis revealed that miR-365 was predicted to bind to NEAT1 (Fig. 2A). To examine the association between NEAT1 and miR-365 in OSCC, miR-365 levels in OSCC tissue and cell lines were determined. As indicated in Fig. 2B, RT-qPCR assay data demonstrated that miR-365 expression was significantly lower in OSCC tissue compared with adjacent non-tumour tissue. In addition, miR-365 expression was significantly reduced in OSCC cell lines compared with hNOK cells (Fig. 2C). It was demonstrated that miR-365 was downregulated in OSCC. In addition, the present study revealed an inverse correlation between NEAT1 and miR-365 expression in OSCC tissue (Fig. 2D), which suggests that reduced miR-365 expression may be involved in OSCC development.

NEAT1 downregulates miR-365 expression via sponging in OSCC cells. As HN4 and Tca-8113 cells exhibited the highest expression levels of NEAT1 among the tested OSCC cell lines, 

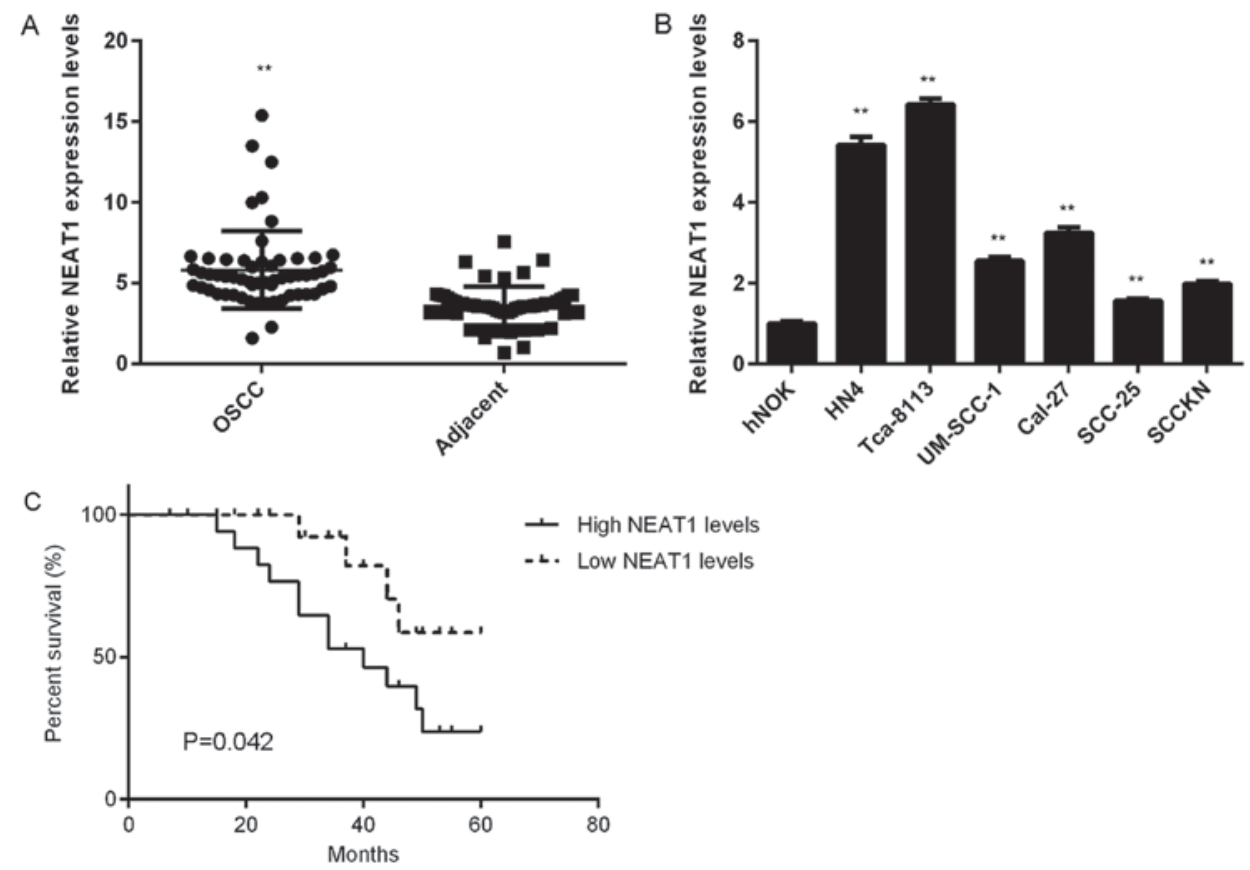

Figure 1. NEAT1 is upregulated in OSCC. (A) RT-qPCR analysis performed to examine NEAT1 levels in OSCC tissue compared with adjacent non-tumor tissue. "* $\mathrm{P}<0.01$ vs. adjacent non-tumor tissue. (B) RT-qPCR performed to examine NEAT1 levels in numerous OSCC cell lines compared with hNOK cells. ${ }^{* *} \mathrm{P}<0.01$ vs. hNOK cells. (C) Patients with OSCC and high NEAT1 expression demonstrated shorter survival time compared with low NEAT1 expression. RT-qPCR, reverse transcription-quantitative polymerase chain reaction; NEAT1, nuclear enriched abundant transcript 1; OSCC, oral squamous cell carcinoma.
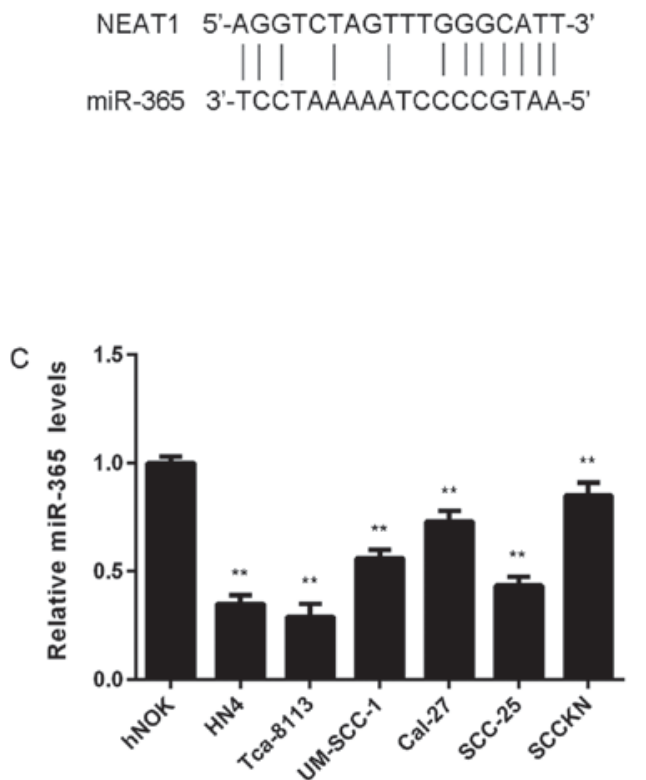

B

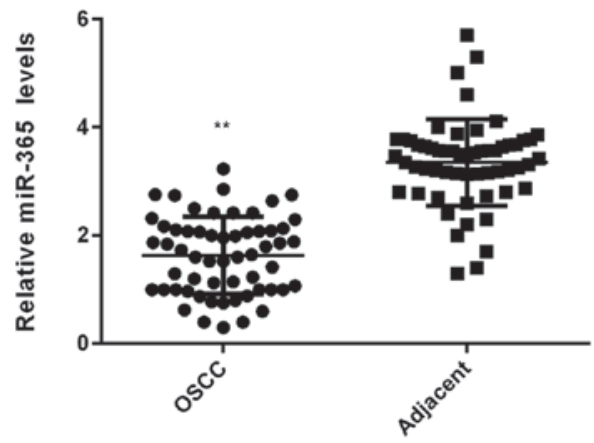

D

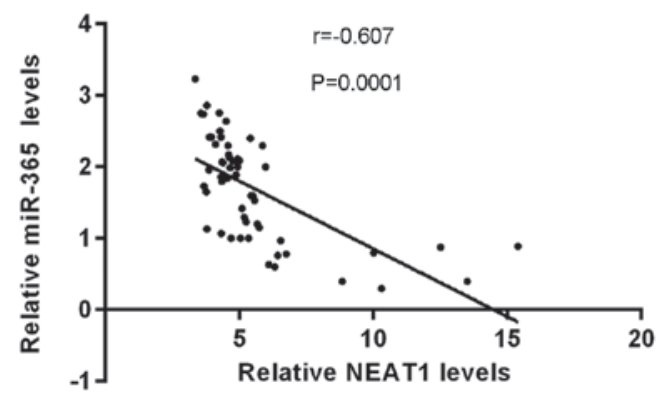

Figure 2. miR-365 is downregulated in OSCC. (A) miR-365 was predicted to bind to NEAT1. (B) RT-qPCR performed to examine miR-365 levels in OSCC tissue compared with adjacent non-tumor tissue. ${ }^{* * *} \mathrm{P}<0.01$ vs. adjacent non-tumor tissue. (C) RT-qPCR was performed to examine miR-365 levels in various OSCC cell lines compared with hNOK cells. ${ }^{* *} \mathrm{P}<0.01$ vs. hNOK cells. (D) Inverse correlation between NEAT1 and miR-365 expression in OSCC tissue. miR-365, microRNA-365; NEAT1, nuclear enriched abundant transcript 1; RT-qPCR, reverse transcription-quantitative polymerase chain reaction; OSCC, oral squamous cell carcinoma; hNOK, human normal oral keratinocytes.

these cell lines were selected for in vitro experiments. To further clarify the association between miR-365 and NEAT1 in HN4 and Tca-8113 cells, a luciferase reporter plasmid containing WT or MT miR-365 binding sites of NEAT1 was constructed in the present study (Fig. 3A). A luciferase reporter gene assay was then performed. The data revealed that transfection with an miR-365 mimic significantly inhibited luciferase activity of WT NEAT1 in OSCC cells but did not affect luciferase activity of MT NEAT1 (Fig. 3B and C). These findings suggest that NEAT1 may be able to sponge miR-365 in OSCC cells. 
A

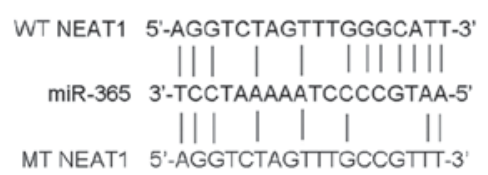

B

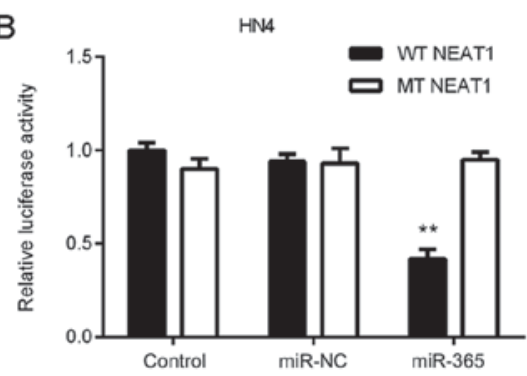

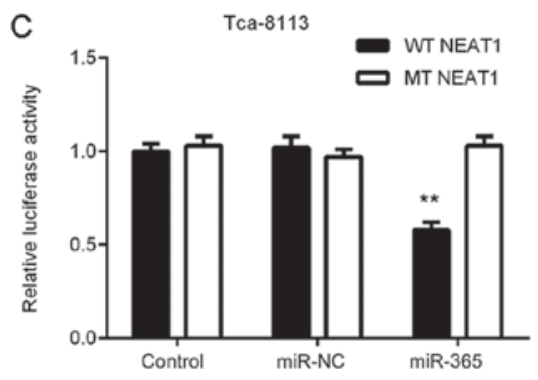

$\mathrm{E}$

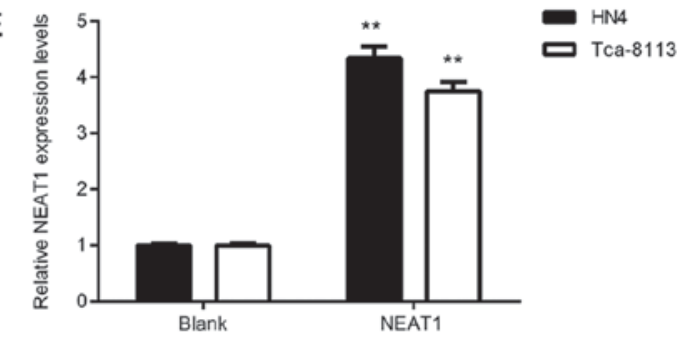

G

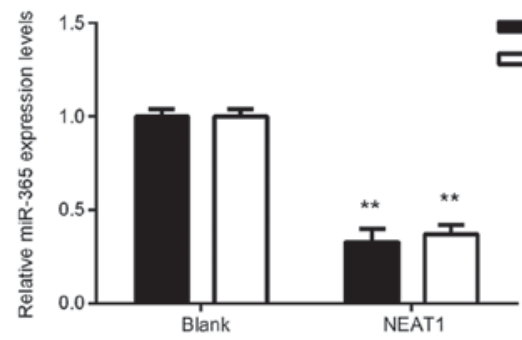

Figure 3. NEAT1 downregulates miR-365 expression via sponging in OSCC cells. (A) Constructed luciferase reporter plasmids containing WT or MT miR-365 binding sites in NEAT1. Luciferase reporter gene assay conducted in (B) HN4 and (C) Tca-8113 cells. Transfection with miR-365 mimic significantly inhibited luciferase activity of WT NEAT1 in OSCC cells, but did not affect the luciferase activity of MT NEAT1. ${ }^{* *} \mathrm{P}<0.01$ vs. Control. HN4 and Tca-8113 cells transfected with (D) NEAT1 siRNA to knock down NEAT1 levels or (E) NEAT1 plasmid to upregulate its expression. (F) NEAT1 overexpression significantly inhibited expression of miR-365 and (G) knock down of NEAT1 enhanced miR-365 expression in HN4 and Tca-8113 cells. "* P<0.01 vs. NC siRNA or Blank. WT, wild-type; MT, mutant type; miR-365, microRNA-365; NEAT1, nuclear enriched abundant transcript 1; OSCC, oral squamous cell carcinoma; siRNA, short interfering RNA; NC, negative control.

Effects of NEAT1 on miR-365 expression of in OSCC cells were evaluated. HN4 and Tca-8113 cells were transfected with NEAT1 siRNA to knock down NEAT1 levels or cells were transfected with NEAT1 plasmid to upregulate its expression. Following transfection, NEAT1 expression was significantly decreased in cells transfected with NEAT1 siRNA compared with the NC siRNA group and was significantly increased in the NEAT1 group compared with the blank control group (Fig. 3D and E). The current study demonstrated that knockdown of NEAT1 enhanced miR-365 expression in OSCC cells and NEAT1 overexpression significantly inhibited miR-365 expression (Fig. $3 \mathrm{~F}$ and $\mathrm{G}$ ). These findings indicated that NEAT1 downregulates miR-365 expression via sponging in OSCC cells.

Knockdown of NEAT1 inhibits OSCC cell invasion. To further study the function of NEAT1 in OSCC cells, wound-healing assays were performed to examine effects of NEAT1 downregulation on OSCC cell migration. As presented in Fig. 4A, migratory capacity of HN4 and Tca-8113 cells was significantly reduced in the NEAT1 siRNA group compared with the NC siRNA group. Transwell assays were performed to evaluate cell invasion. As presented in Fig. 4B, invasiveness of HN4 and Tca-8113 cells in the NEAT1 siRNA group was significantly reduced following inhibition of NEAT1 expression. Consistently, western blot data indicated that protein expression of MMP2 and MMP9, two key enzymes involved in tumour cell invasiveness (22), was significantly reduced in the NEAT1 siRNA group compared with the NC siRNA group (Fig. 4C). These findings indicate that knockdown of NEAT1 expression inhibits OSCC cell migration and invasion.

Inhibition of NEAT1 suppresses OSCC cell migration and invasion by sponging miR-365. Based on the aforementioned findings, it was speculated that NEAT1 may regulate migration and invasiveness of OSCC cells by sponging miR-365. To clarify this, NEAT1 siRNA-transfected OSCC cells were co-transfected with $\mathrm{NC}$ inhibitor or miR-365 inhibitor. Following co-transfection, miR-365 levels were significantly reduced in the NEAT1 siRNA+miR-365 inhibitor group compared with the NEAT1 siRNA+NC inhibitor group (Fig. 5A). Further investigation indicated that migration ability and invasiveness of OSCC cells was increased in the NEAT1 siRNA+miR-365 inhibitor group compared with the NEAT1 siRNA+NC inhibitor group (Fig. 5B and C). Consistently, suppressive effects of NEAT1 inhibition on MMP2 and MMP9 protein expression were reversed following co-transfection with miR-365 inhibitor (Fig. 5D). These findings demonstrated 
A
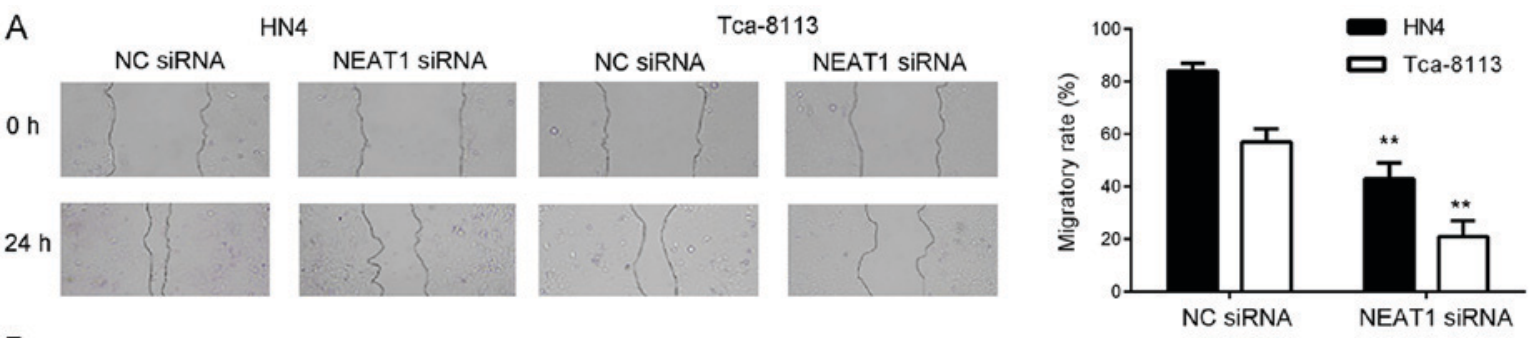

B
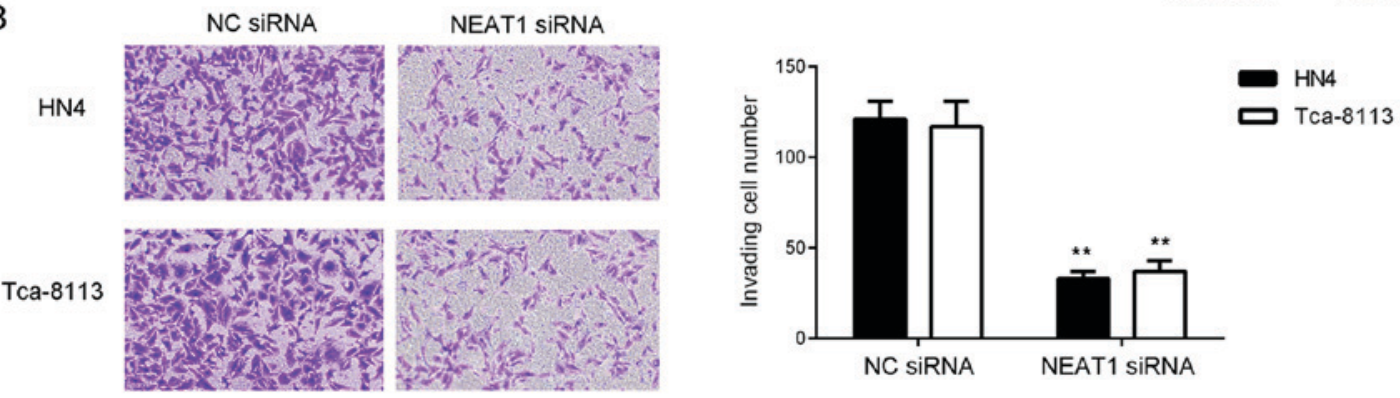

C
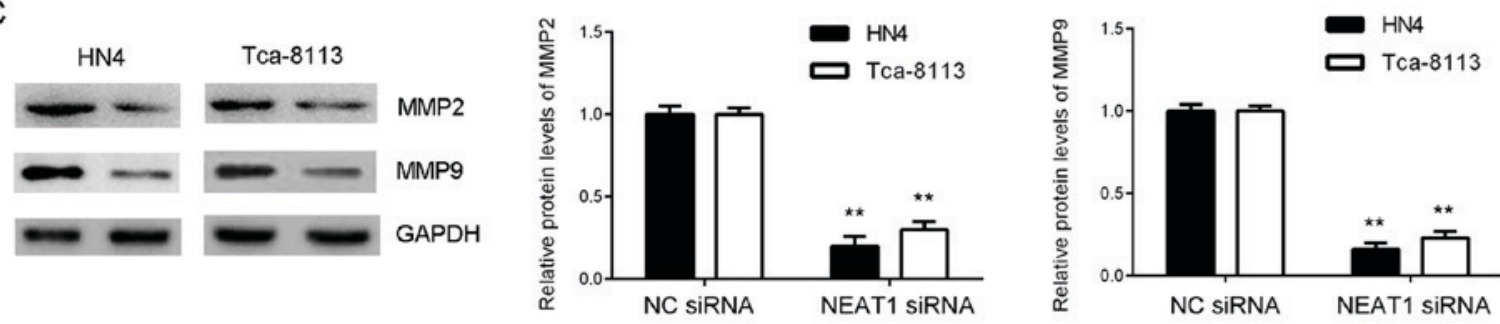

Figure 4. Knockdown of NEAT1 inhibits OSCC cell invasion. (A) Wound healing (magnification, x40) and (B) Transwell assays performed to study effects of NEAT1 downregulation on HN4 and Tca-8113 cell migration and invasion (magnification, x400). (C) Western blot assay to examine MMP2 and MMP9 protein expression. ${ }^{* *} \mathrm{P}<0.01$ vs. NC siRNA. NEAT1, nuclear enriched abundant transcript 1; siRNA, short interfering RNA; MMP, matrix metalloproteinase; NC, negative control.

that inhibition of NEAT1 suppressed OSCC migration and invasiveness via inhibition of miR-365 sponging.

\section{Discussion}

To the best of our knowledge, the function and regulatory mechanism of NEAT1 in OSCC has not been reported previously. The present study revealed that NEAT1 was significantly upregulated in OSCC tissue and cell lines and that its upregulation was significantly associated with advanced clinical stage and shorter survival time of patients with OSCC. Data from luciferase reporter gene assays confirmed an interaction between miR-365 and NEAT1 and demonstrated that NEAT1 negatively regulated miR-365 expression in two OSCC cell lines. Furthermore, inhibition of NEAT1 expression led to a significant decrease in OSCC cell migration and invasion, whereas inhibition of miR-365 eliminated NEAT1 knockdown-induced suppressive effects on OSCC cell migration and invasion. In addition, miR-365 was significantly downregulated in OSCC tissue and cell lines and an inverse correlation was observed between miR-365 and NEAT1 expression in OSCC tissue.

Previous studies have demonstrated that NEAT1 functions as an oncogene in multiple types of human cancer $(24,25)$. For instance, NEAT1 promotes laryngeal squamous cell cancer through regulation of the miR-107/CDK6 signalling pathway (24). NEAT1 is an unfavourable prognostic factor in gastric cancer, as it promotes tumour cell migration and invasion (25). However, the role of NEAT1 in OSCC remains unclear. In the present study, it was demonstrated that NEAT1 expression was significantly higher in OSCC tissue and cell lines compared with adjacent non-tumour tissue and normal oral keratinocytes, respectively. Additionally, upregulation of NEAT1 may be associated with lymph node metastasis, advanced clinical stage and poor prognosis of patients with OSCC. Similarly, increased NEAT1 expression was further associated with unfavourable clinical characteristics and poor prognosis in ovarian cancer (26), oesophageal squamous cell carcinoma (27), gastric cancer (28) and liver cancer (29).

The present study investigated underlying regulatory mechanisms of NEAT1 in OSCC metastasis. A bioinformatics analysis predicted that miR-365 is a potential target of NEAT1. Luciferase reporter gene assays revealed that transfection with a miR-365 mimic significantly inhibited luciferase activity of WT NEAT1 in OSCC cells but did not affect luciferase activity of MT NEAT1, which indicates that NEAT1 may sponge miR-365 in OSCC cells. To further study the relationship between miR-365 and NEAT1 in OSCC, experiments that demonstrated that miR-365 was significantly downregulated in OSCC tissue and cell lines were performed. The current study observed an inverse correlation between miR-365 and NEAT1 in OSCC tissue.

The function of NEAT1 in OSCC metastasis was examined in vitro and it was revealed that knockdown of NEAT1 reduced OSCC cell migration and invasion. As miR-365 expression was downregulated by NEAT1 in OSCC cells, it was speculated 
A

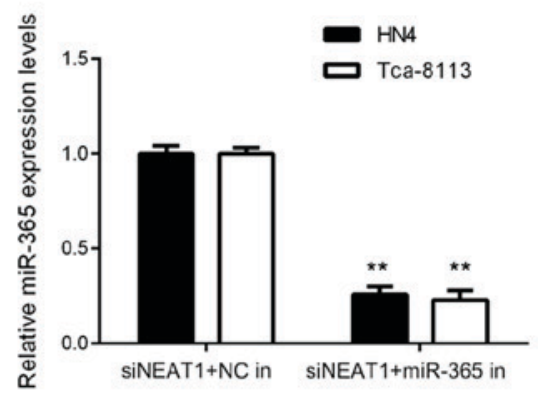

B HN4 Tca-8113
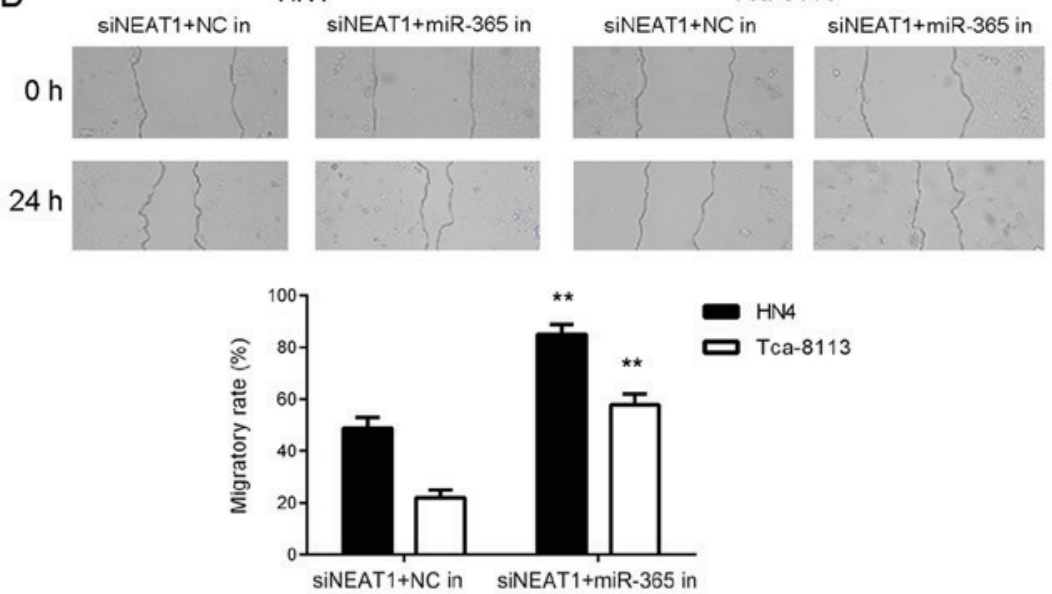

C

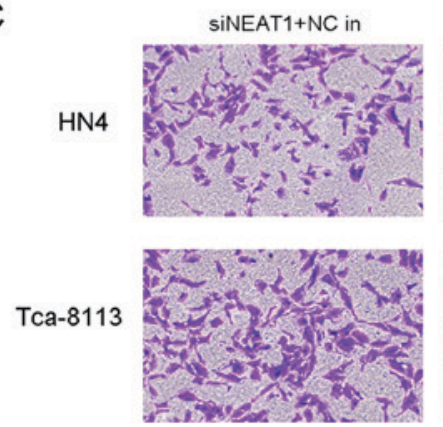

SiNEAT1+miR-365 in
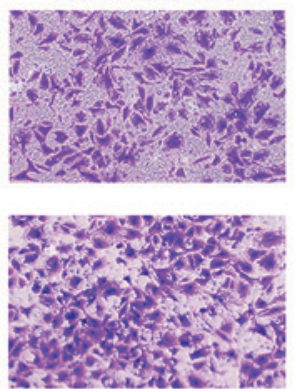

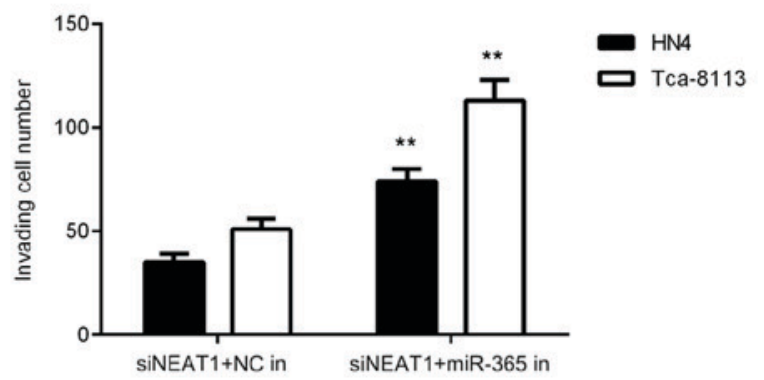

D
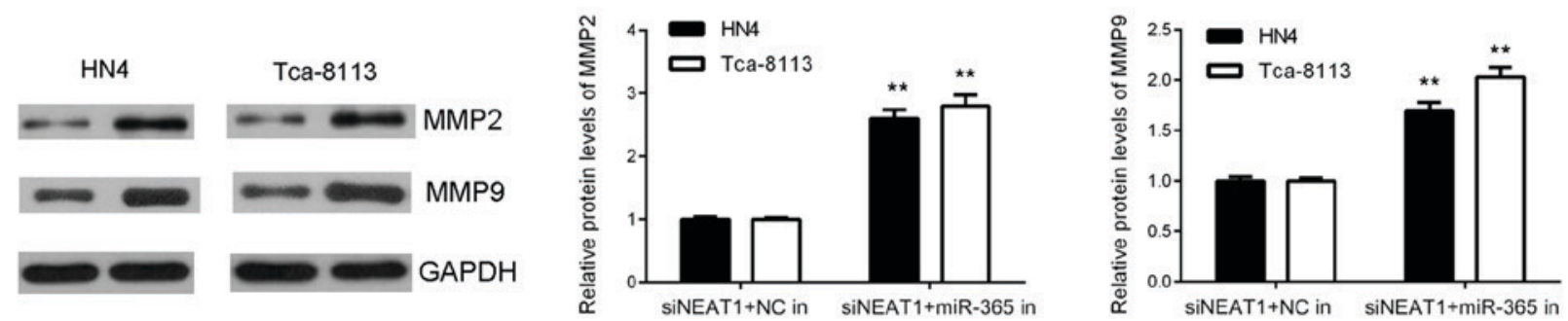

Figure 5. Inhibition of NEAT1 suppresses OSCC cell migration and invasion by sponging miR-365. NEAT1 siRNA transfected OSCC cells co-transfected with NC or miR-365 inhibitor. (A) Reverse transcription-quantitative polymerase chain reaction analysis of miR-365 levels. (B) Wound healing (magnification, $\mathrm{x} 40$ ) and (C) Transwell assay performed to examine cell migration and invasion (magnification, x400). (D) Western blot analysis of MMP2 and MMP9 protein expression. ${ }^{* *} \mathrm{P}<0.01$ vs. siNEAT1+NCin. NEAT1, nuclear enriched abundant transcript 1; siRNA, short interfering RNA; OSCC, oral squamous cell carcinoma; NC, negative control; miR-365, microRNA-365; MMP, matrix metalloproteinase; NC, negative control; in, inhibitor.

that miR-365 may be involved in NEAT1-mediated OSCC cell migration and invasion. Experimental data confirmed suppressive effects of NEAT1 inhibition on OSCC cell migration and invasion, as well as downregulation of MMP2 and MMP9 protein expression.

To the best of our knowledge, this is the first study to report that NEAT1 promotes migration and invasiveness of OSCC cells by sponging miR-365. Therefore, the current study suggests that NEAT1 may be used as a novel therapeutic target when treating patients with OSCC.

\section{Acknowledgements}

Not applicable.

\section{Funding}

No funding was received.

\section{Availability of data and materials}

All data generated or analyzed during this study are included in this published article.

\section{Authors' contributions}

XL wrote the manuscript and performed the experiments. WS collected clinical samples and performed the statistical analysis. FZ designed the present study and revised the manuscript. All authors read and approved the final manuscript.

\section{Ethics approval and consent to participate}

The present study was approved by the Medical Ethics Committee of Stomatological Hospital of Jinan City (Jinan, China). All patients provided written informed consent. 


\section{Patient consent for publication}

Not applicable.

\section{Competing interests}

The authors declare that they have no competing interests.

\section{References}

1. Shan Z, Yang G, Xiang W, Pei-Jun W and Bin Z: Effects of resveratrol on oral squamous cell carcinoma (OSCC) cells in vitro. J Cancer Res Clin Oncol 140: 371-374, 2014.

2. Lu L, Xue X, Lan J, Gao Y, Xiong Z, Zhang H, Jiang W, Song W and Zhi Q: MicroRNA-29a upregulates MMP2 in oral squamous cell carcinoma to promote cancer invasion and anti-apoptosis. Biomed Pharmacother 68: 13-19, 2014.

3. Xu R, Zeng G, Gao J, Ren Y, Zhang Z, Zhang Q, Zhao J, Tao H and Li D: MiR-138 suppresses the proliferation of oral squamous cell carcinoma cells by targeting Yes-associated protein 1. Onco Rep 34: 2171-2178, 2015.

4. Tan DS, Wang W, Leong HS, Sew PH, Lau DP, Chong FT, Krisna SS, Lim TK and Iyer NG: Tongue carcinoma infrequently harbor common actionable genetic alterations. BMC Cancer 14 679,2014

5. Shi Z and Stack MS: Molecules of cell adhesion and extracellular matrix proteolysis in oral squamous cell carcinoma. Histol Histopathol 25: 917-932, 2010.

6. Zhu J, Shi H, Liu H, Wang X and Li F: Long non-coding RNA TUG1 promotes cervical cancer progression by regulating the miR-138-5p-SIRT1 axis. Oncotarget 8: 65253-65264, 2017.

7. Zhang Y, Dai Q, Zeng F and Liu H: MALAT1 promotes the proliferation and metastasis of osteosarcoma cells by activating the Rac1/JNK pathway via targeting MiR-509. Oncol Res: Apr 27, 2018 (Epub ahead of print).

8. Zhang JJ, Wang DD, Du CX and Wang Y: Long noncoding RNA ANRIL promotes cervical cancer development by acting as a sponge of miR-186. Oncol Res: May 22, 2017 (Epub ahead of print).

9. Wang S, Hui Y, Li X and Jia Q: Silencing of lncRNA-CCDC26 restrains the growth and migration of glioma cells in vitro and in vivo via targeting miR-203. Oncol Res: Jun 9, 2017 (Epub ahead of print).

10. Zhu G, Wang S, Chen J, Wang Z, Liang X, Wang X, Jiang J, Lang $J$ and Li L: Long noncoding RNA HAS2-AS1 mediates hypoxia-induced invasiveness of oral squamous cell carcinoma. Mol Carcinog 56: 2210-2222, 2017.

11. Fang Z, Zhao J, Xie W, Sun Q, Wang H and Qiao B: LncRNA UCA1 promotes proliferation and cisplatin resistance of ora squamous cell carcinoma by sunppressing miR-184 expression. Cancer Med 6: 2897-2908, 2017.

12. Yan G, Wang X, Yang M, Lu L and Zhou Q: Long non-coding RNA TUG1 promotes progression of oral squamous cell carcinoma through upregulating FMNL2 by sponging miR-219. Am J Cancer Res 7: 1899-1912, 2017

13. Liu Z, Wu C, Xie N and Wang P: Long non-coding RNA MEG3 inhibits the proliferation and metastasis of oral squamous cell carcinoma by regulating the $\mathrm{WNT} / \beta$-catenin signaling pathway. Oncol Lett 14: 4053-4058, 2017.
14. Li S, Yang J, Xia Y, Fan Q and Yang KP: Long noncoding RNA NEAT1 promotes proliferation and invasion via targeting miR-181a-5p in non-small cell lung cancer. Oncol Res 26: 289-296, 2018.

15. Chen Q, Cai J, Wang Q, Wang Y, Liu M, Yang J, Zhou J, Kang C, Li $\mathrm{M}$ and Jiang $\mathrm{C}$ : Long noncoding RNA NEAT1, regulated by the EGFR pathway, contributes to glioblastoma progression through the WNT/ $\beta$-Catenin pathway by scaffolding EZH 2 . Clin Cancer Res 24: 684-695, 2018.

16. An J, Lv W and Zhang Y: LncRNA NEAT1 contributes to paclitaxel resistance of ovarian cancer cells by regulating ZEB1 expression via miR-194. Onco Targets Ther 10: 5377-5390, 2017.

17. Ambros V: The functions of animal microRNAs. Nature 431: 350-355, 2004.

18. Lu J, Getz G, Miska EA, Alvarez-Saavedra E, Lamb J, Peck D, Sweet-Cordero A, Ebert BL, Mak RH, Ferrando AA, et al: MicroRNA expression profiles classify human cancers. Nature 435: 834-838, 2005.

19. Zhou Y, Yang C, Wang K, Liu X and Liu Q: MicroRNA-33b inhibits the proliferation and migration of osteosarcoma cells via targeting hypoxia-inducible factor-1 $\alpha$. Oncol Res 25: 397-405, 2017.

20. Wang $\mathrm{Y}, \mathrm{Xu} \mathrm{C}$ and Zhang $\mathrm{X}$ : MicroRNA-365 inhibits ovarian cancer progression by targeting Wnt5a. Am J Cancer Res 7: 1096-1106, 2017

21. Zhu Y, Zhao H, Rao M and Xu S: MicroRNA-365 inhibits proliferation, migration and invasion of glioma by targeting PIK3R3. Oncol Rep 37: 2185-2192, 2017.

22. Pietruszewska W, Bojanowska-Poźniak K and Kobos J: Matrix metalloproteinases MMP1, MMP2, MMP9 and their tissue inhibitors TIMP1, TIMP2, TIMP3 in head and neck cancer: An immunohistochemical study. Otolaryngol Pol 70: 32-43, 2016.

23. Livak KJ and Schmittgen TD: Analysis of relative gene expression data using real-time quantitative PCR and the 2(-Delta Delta C(T)) method. Methods 25: 402-408, 2001.

24. Wang $\mathrm{P}, \mathrm{Wu} \mathrm{T}$, Zhou H, Jin Q, He G, Yu H, Xuan L, Wang X, Tian L, Sun Y, et al: Long noncoding RNA NEAT1 promotes laryngeal squamous cell cancer through regulating miR-107/CDK6 pathway. J Exp Clin Cancer Res 35: 22, 2016.

25. Fu JW, Kong Y and Sun X: Long noncoding RNA NEAT1 is an unfavorable prognostic factor and regulates migration and invasion in gastric cancer. J Cancer Res Clin Oncol 142: 1571-1579, 2016.

26. Chen ZJ, Zhang Z, Xie BB and Zhang HY: Clinical significance of up-regulated lncRNA NEAT1 in prognosis of ovarian cancer. Eur Rev Med Pharmacol Sci 20: 3373-3377, 2016.

27. Chen X, Kong J, Ma Z, Gao S and Feng X: Up regulation of the long non-coding RNA NEAT1 promotes esophageal squamous cell carcinoma cell progression and correlates with poor prognosis. Am J Cancer Res 5: 2808-2815, 2015.

28. Ma Y, Liu L, Yan F, Wei W, Deng J and Sun J: Enhanced expression of long non-coding RNA NEAT1 is associated with the progression of gastric adenocarcinomas. World J Surg Oncol 14: 41, 2016.

29. Liu Z, Chang Q, Yang F, Liu B, Yao HW, Bai ZG, Pu CS, Ma XM, Yang Y, Wang TT, et al: Long non-coding RNA NEAT1 overexpression is associated with unfavorable prognosis in patients with hepatocellular carcinoma after hepatectomy: A Chinese population-based study. Eur J Surg Oncol 43: 1697-1703, 2017.

This work is licensed under a Creative Commons Attribution-NonCommercial-NoDerivatives 4.0 International (CC BY-NC-ND 4.0) License. 